\title{
Genetic and clinical characteristics of primary and secondary glioblastoma is associated with differential molecular subtype distribution
}

\author{
Rui Li ${ }^{1, *}$, Hailin Li ${ }^{1, *}$, Wei Yan ${ }^{1}$, Pei Yang ${ }^{2,4}$, Zhaoshi Bao ${ }^{2,4}$, Chuanbao Zhang ${ }^{2,4}$, Tao \\ Jiang ${ }^{2,3,4}$ and Yongping You ${ }^{1}$ \\ ${ }^{1}$ Department of Neurosurgery, The First Affiliated Hospital of Nanjing Medical University, Nanjing, China \\ 2 Beijing Neurosurgical Institute, Capital Medical University, Beijing, China \\ ${ }^{3}$ Beijing Institute for Brain Disorders Brain, Tumor Center, Beijing, China \\ ${ }^{4}$ Department of Neurosurgery, Beijing Tiantan Hospital, Capital Medical University, Beijing, China \\ * These authors have contributed equally to this work \\ Correspondence to: Yongping You, email: yypl9@njmu.edu.cn \\ Keywords: Primary glioblastomas, Secondary glioblastomas, Molecular subtypes, Whole transcriptome sequencing \\ Received: December 05, $2014 \quad$ Accepted: January 21,2015 Published: January 31, 2015
}

This is an open-access article distributed under the terms of the Creative Commons Attribution License, which permits unrestricted use, distribution, and reproduction in any medium, provided the original author and source are credited.

\section{ABSTRACT}

Glioblastoma multiforme (GBM) is classified into primary (PGBM) or secondary (sGBM) based on clinical progression. However, there are some limits to this classification for insight into genetically and clinically distinction between pGBM and SGBM. The aim of this study is to characterize pGBM and SGBM associating with differential molecular subtype distribution. Whole transcriptome sequencing data was used to assess the distribution of molecular subtypes and genetic alterations in 88 pGBM and 34 sGBM in a Chinese population-based cohort, and the biological progression and prognostic impact were analyzed by combining clinical information. Forty-one percentage of pGBM were designated as Mesenchymal subtype, while only $15 \%$ were the Proneural subtype. However, sGBM displayed the opposite ratio of Mesenchymal (15\%) and Proneural (44\%) subtypes. Mutations in isocitrate dehydrogenase-1 (IDH1) were found to be highly concentrated in the Proneural subtypes. In addition, patients with SGBM were 10 years younger on average than those with pGBM, and exhibited clinical features of shorter overall survival and frontal lobe tumor location tendency. Furthermore, in SGBM, gene sets related to malignant progression were found to be enriched. Overall, these results reveal the intrinsic distinction between PGBM and SGBM, and provide insight into the genetic and clinical attributes of GBM.

\section{INTRODUCTION}

Glioblastoma multiforme (GBM) is the most lethal type of adult brain tumor, accounting for $60-70 \%$ of all gliomas. Despite the advanced treatment, the median survival of patients with GBM is approximately 15 months [1]. Clinically, GBM is divided into primary glioblastoma (pGBM), which progresses rapidly and has an absence of precursor lesions, and secondary glioblastoma (sGBM), which progresses as diffuse astrocytoma (WHO grade II) or anaplastic astrocytoma (WHO grade III) [2-4]. Although pGBM and sGBM display distinct clinical progression, they are histologically indistinguishable. For further insight, research efforts have focused on investigating GBM molecular profiles. Recent studies suggest that isocitrate dehydrogenase-1 (IDH1) mutations, which are frequently detected in sGBM $(>80 \%)$ but are rare in pGBM $(<5 \%)$, may be considered as a diagnostic molecular biomarker of sGBM [5-9]. The Cancer Genome Atlas (TCGA) Research Network described a robust gene expression-based molecular classification of GBM into Proneural, Neural, Classical and Mesenchymal subtypes [10].

In the present study, whole transcriptome sequencing 
Table 1: Clinical features of patients with pGBM and sGBM according to their molecular subtypes.

\begin{tabular}{|c|c|c|c|c|c|c|c|c|}
\hline & \multicolumn{2}{|c|}{ Proneural } & \multicolumn{2}{c|}{ Neural } & \multicolumn{2}{c|}{ Classical } & \multicolumn{2}{c|}{ Mesenchymal } \\
\hline & pGBM & sGBM & pGBM & sGBM & pGBM & sGBM & pGBM & sGBM \\
\hline No. of patients & 13 & 15 & 7 & 3 & 32 & 11 & 36 & 5 \\
\hline Age & & & & & & & & \\
\hline$<50$ years & 10 & 13 & 2 & 0 & 15 & 9 & 12 & 2 \\
\hline$\geq 50$ years & 3 & 2 & 5 & 3 & 17 & 2 & 24 & 3 \\
\hline Gender & & & & & & & & 26 \\
\hline Male & 6 & 13 & 3 & 1 & 20 & & 3 \\
\hline Female & 7 & 2 & 4 & 2 & 12 & 4 & 10 & 2 \\
\hline Location & & & & & & & & \\
\hline Frontal lobe & 5 & 9 & 3 & 3 & 12 & 6 & 9 & 5 \\
\hline Temporal lobe & 6 & 1 & 1 & 0 & 9 & 0 & 14 & 0 \\
\hline
\end{tabular}

data was analyzed to characterize the distribution of molecular subtypes in 88 pGBM and 34 sGBM from a Chinese population-based cohort. Both pGBM and sGBM samples were analyzed for the presence of biomarkers and enriched gene sets. The clinical features of the patients with pGBM and sGBM were assessed, including overall survival time and tumor location. The results suggest that different clinical and genetic profiles of pGBM and sGBM mainly result from the different proportions of the four molecular subtypes in them.

\section{RESULTS}

\section{Distribution of molecular subtypes and gene alterations in pGBM and sGBM}

As shown in Figure 1 and Table 1, among 88 pGBM, 36 cases $(41 \%)$ were Mesenchymal subtype, while only 13 cases $(15 \%)$ were Proneural subtype. However, sGBM showed the opposite ratio of Mesenchymal (5/34, 15\%) and Proneural $(15 / 34,44 \%)$ subtypes. The proportion of
Neural subtypes in pGBM (8\%) and sGBM (9\%) were similar. In addition, $36 \%$ of pGBM were classified as Classical subtype, which was slightly higher than $32 \%$ of sGBM.

With respect to gene signatures, the frequency of IDH1 mutation in SGBM was 53\%, nearly four times as high as that of pGBM (14\%). Furthermore, the majority of IDH1 mutations were clustered in Proneural subtypes in both pGBM and sGBM, whereas IDH2 mutation was absent in the whole cohort. TP53 and IDH1 mutations were mutually exclusive in pGBM, however this was not the case in SGBM. Epidermal growth factor receptor (EGFR) mutation was detected in $35 \%$ of sGBM and $26 \%$ of pGBM. Furthermore, Alpha Thalassemia/Mental Retardation Syndrome X-linked (ATRX) mutation was detected in $10 \%$ of pGBM, but was absent in sGBM. Such alteration was mutually exclusive with IDH1 mutation and EGFR mutation, but co-occurred with TP53 mutation in pGBM.

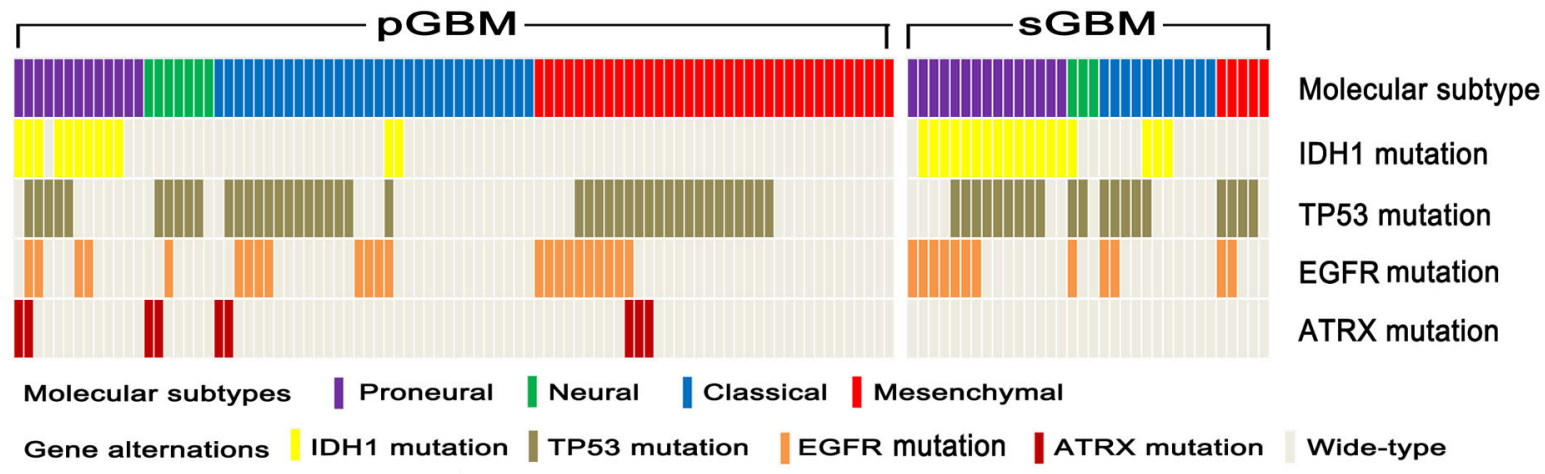

Figure 1: Distribution of molecular subtypes and genetic alteration signatures in pGBM and sGBM. Distribution and correlation between GBM molecular subtypes (Proneural, Neural, Classical and Mesenchymal), IDH1 mutation, TP53 mutation, EGFR mutation and ATRX mutation in pGBM and sGBM. Molecular subtypes and genetic alterations are indicated in different colors. 


\section{Gene set enrichment analysis for pGBM and sGBM}

Given the data of whole transcriptome sequencing of 88 primary glioblastomas and 34 secondary glioblastomas, we performed Gene set enrichment analysis (GSEA) and got results that gene sets related to defense response, inflammatory response and locomotory behavior were significantly enriched in the primary glioblastomas $(\mathrm{P}<0.001)$, while chromosome organization, cell cycle, mRNA processing and mitosis gene sets were clustered in secondary glioblastomas $(\mathrm{P}<0.001)$. (Figure 2 and Table S1)

\section{Prognostic impact of combined analysis of pGBM and SGBM molecular subtypes}

The median overall survival of all patients with pGBM after diagnosis was 381 days, whereas the median overall survival was 284 days in patients with sGBM (Figure 3A). As shown in Figure 3B, patients carrying the IDH1 mutation experienced an improved prognosis (1074 days for pGBM and 346 days for sGBM) compared with patients who did not have such a mutation (372 days for pGBM and 256 days for sGBM). When this analysis was combined with molecular subtypes, patients with Neural subtype pGBM exhibited the longest overall survival, followed by patients with Proneural subtype pGBM, with 970 days of median overall survival. Patients with Mesenchymal and Proneural subtypes of sGBM resulted in the worst clinical outcome, with survival of 236 and 231 days, respectively (Figure 3C).

\section{Clinical features of pGBM and sGBM}

As shown in Figure 4, GBM predominantly affected males in this study, with a male to female ratio of 1.67 in pGBM and 2.4 in sGBM. With respect to anatomical localization, the frontal and temporal lobe were the most commonly involved sites, with $68 \%$ of sGBM located in the frontal lobe, while only one case involved the temporal lobe. A similar phenomenon was observed in Proneural (45\%), Classical (38\%) and Neural (58\%) subtypes. However, among Mesenchymal subtypes, the temporal lobe was found to be the predominant site (41\%). pGBM show widespread anatomical distribution and tumors were more commonly located in the left hemisphere of the brain. The mean age of patients diagnosed with sGBM was $39.26 \pm 2.05$ years, whereas the mean age of patients diagnosed with pGBM was $49.61 \pm 1.35$ years. For the molecular subtypes, the cohort with the oldest age of diagnosis was Mesenchymal subtype (52.06 \pm 1.64 years), followed by Neural ( $50.75 \pm 3.07$ years), Classical
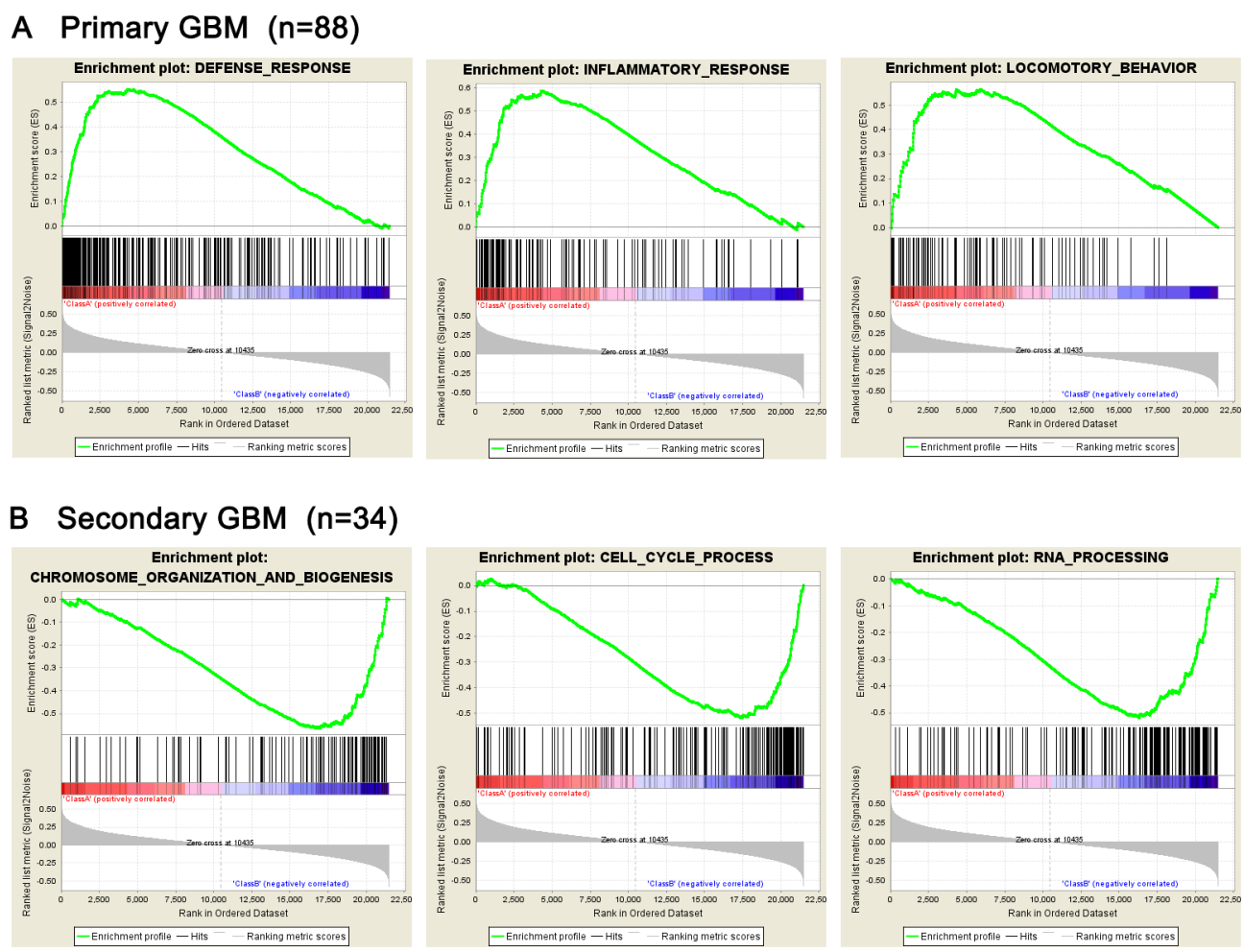

Figure 2: Presence of gene sets related to biological processes analyzed by GSEA. (A) Gene sets related to biological processes in $\mathrm{pGBM}$; (B) Gene sets related to biological processes in sGBM. 
$(45.50 \pm 1.92$ years) and Proneural $(39.48 \pm 1.65$ years $)$ The age distribution of the four molecular subtypes in pGBM and sGBM was further analyzed, and patients with Classical subtype sGBM were found to be significantly younger than those with same subtype in pGBM (mean age of 36.45 versus 47.97 years; $P=0.0125$ ). This trend was also observed for Mesenchymal subtype (46.60 years for sGBM versus 53.89 for pGBM $[P=0.2241])$, and Neural subtype (41.00 years for sGBM versus 54.43 years for pGBM $[P=0.0934])$, whereas Proneural subtypes had similar age of diagnosis (38.53 years for sGBM versus 39.23 years for $\mathrm{pGBM}[P=0.8552])$.

\section{DISCUSSION}

GBM is the most common and lethal type of glioma in adults with an overall survival of less than two years [11-14]. Clinically, GBM is categorized into pGBM and
sGBM based on malignant progression. Although they are histologically indistinguishable, pGBM and sGBM can be identified by characterized biomarkers reported in previous studies [7, 11, 15-18].

In the present study, 122 GBM were characterized, including 88 pGBM and 34 sGBM, based on data from whole transcriptome sequencing and clinical information. Each sample was classified into a molecular subtype and found to exhibit different proportions in two histological types of GBM. Approximately $44 \%$ of sGBM were classified as Proneural subtypes, which was significantly higher than the proportion of this subtype in pGBM, but not as high as the frequency reported in other studies $[8,10]$. In addition, among 15 sGBM with Proneural signature, 14 tumors (93\%) carried IDH1 gene mutation. This high frequency of IDH1 mutation was also found in Proneural pGBM, amounting to $77 \%$ (10/13), which is higher than the $30 \%$ reported in previous studies by Verhaak and colleagues [10] This observation suggests
A

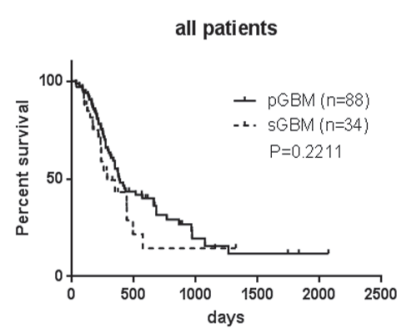

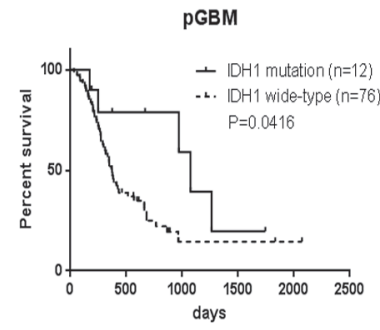
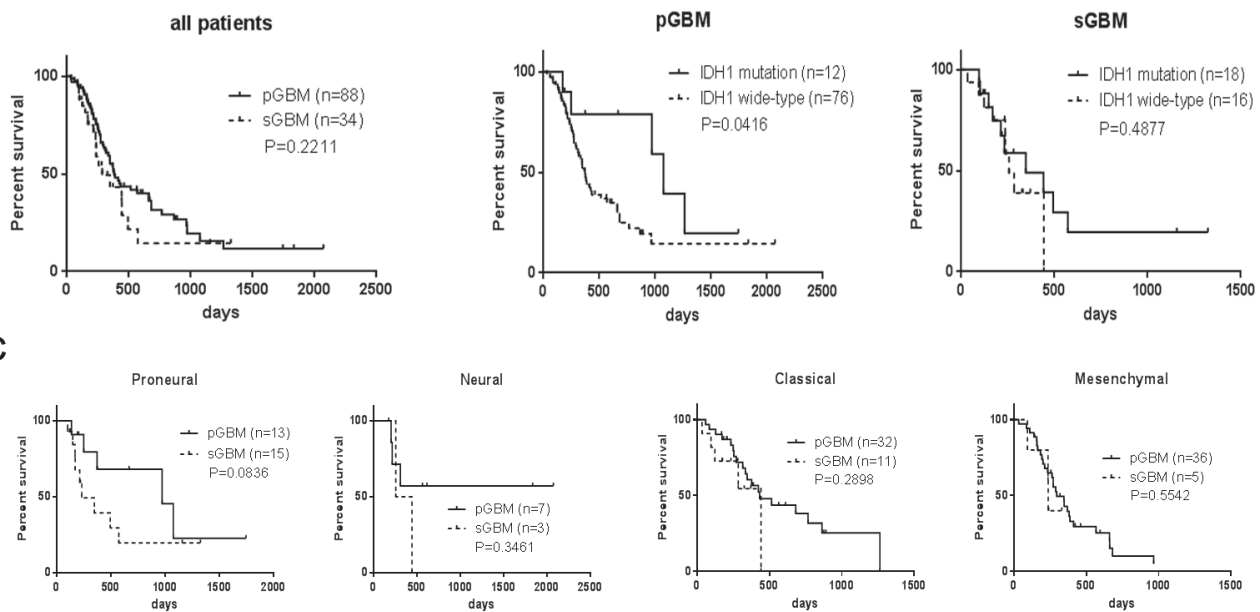
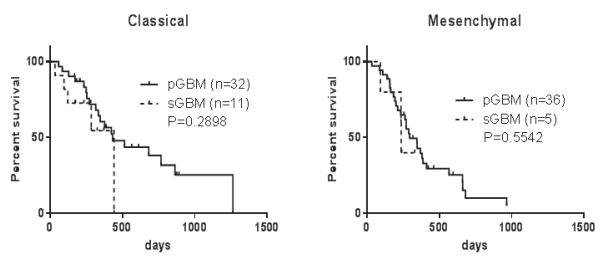

Figure 3: Kaplan-Meier analysis of overall survivals of patients with GBM. (A) Overall survival of patients with pGBM and sGBM; (B) Overall survivals of patients with or without IDH1 mutation in pGBM and sGBM; (C) Overall survivals of patients with Proneural, Neural, Classical and Mesenchymal subtypes.

A
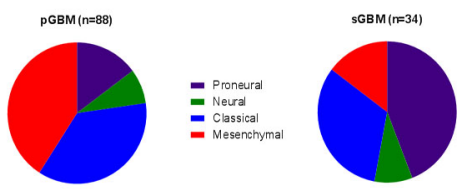

B

B all patients
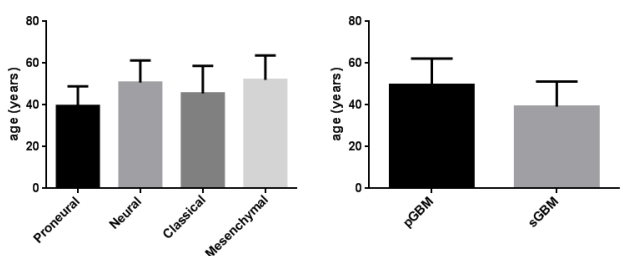

C
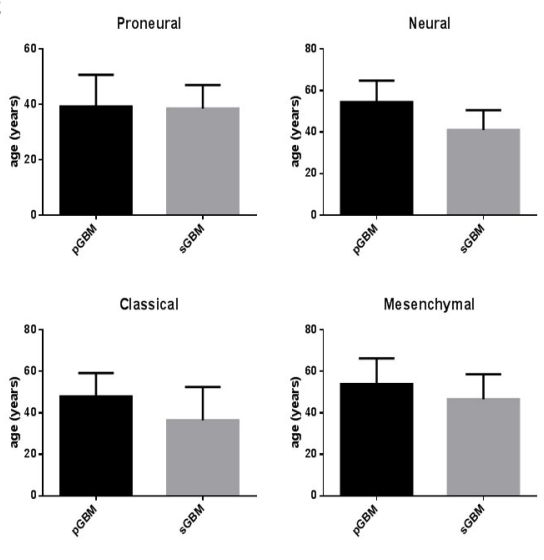

Figure 4: Age distribution of patients with GBM. (A) Distribution of molecular subtypes in pGBM and sGBM; (B) Age distribution of patients with molecular and clinical subtypes of GBM; (C) Age distribution of patients with four molecular subtypes of GBM. *, P $<0.05$; $* *, \mathrm{P}<0.01 ; * * * *, \mathrm{P}<0.0001$. 
that IDH1 mutation might characterize the Proneural subtype, however this cannot be considered as a definitive marker for sGBM perfectively. From the perspective of molecular subtypes rather than the clinical evaluation, the differences between $\mathrm{pGBM}$ and $\mathrm{sGBM}$ resulted from the distinct distribution of four molecular subtypes, especially the inverse ratio between Proneural and Mesenchymal subtypes observed in pGBM and sGBM. The distribution of molecular subtypes was also found to impact the survival of patients with pGBM and sGBM. In the cohort analyzed in this study, patients carrying an IDH1 mutation in pGBM exhibited approximately three times longer survival than those without such mutations. However, such advantage of IDH1 mutation on prognostic impact was not observed in sGBM. One would expect that the Mesenchymal subtype, which had the worst prognosis in terms of length of survival, would occupy high frequency in primary glioblastoma.

The ATRX plays an important role in telomere homeostasis via regulating incorporation of histone variant H3.3 into telomeric chromatin [19-21]. ATRX mutations were recently identified in $7 \%$ of pGBM and more than half of sGBM and were associated with an alternative lengthening of telomeres (ALT) phenotype among GBM $[22,23]$. In the present study, ATRX mutations were detected in 9 of $88(10 \%)$ pGBM, but were absent in sGBM. Furthermore, ATRX mutation co-occurred with TP53 mutation, but was mutually exclusive with IDH1/2 mutation, which is contrary to previous reports $[22,23]$. Ethnic and racial disparities, as well as analysis methods may be factors for such differences.

Similar to previous studies, patients diagnosed with sGBM were 10 years younger than patients with pGBM in this cohort $[12,13]$. Notably, when analyzed in molecular subtypes, patients with Proneural were 5 years younger than patients with Classical subtypes and 10 years younger than Neural and Mesenchymal subtypes patients. Except for the Classical subtype, there was no significant difference in age between patients with pGBM and sGBM in the three other subtypes. In addition, Proneural and Classical subtypes made up $76 \%$ of sGBM patients in this study. These findings verified our hypothesis that different molecular subtype distribution could cause the phenomenon of patients with sGBM being 10 years younger than those with pGBM.

The results from this study suggest that sGBM is predominantly located in the frontal lobe, which is consistent with a previous study by Lai and colleagues [24]. Contrary to sGBM, there was no preferable anatomical cluster location among pGBM. Further combined analysis with whole transcriptome sequencing data revealed that most Neural subtype GBM are located in the frontal lobe, whereas Mesenchymal subtypes are predominantly located in the temporal lobe. Proneural and Classical subtypes were found to be more likely located in the frontal lobe than the temporal lobe. This finding suggests that the predominance of frontal lobe involvement with sGBM partly results from the high frequency of Mesenchymal subtype and rare Proneural subtype in sGBM, which also verified our hypothesis that clinical distinction of $\mathrm{pGBM}$ and sGBM glioblastoma was associated with differential molecular subtype distribution.

GSEA was performed for pGBM and sGBM in this study, with enriched gene sets related to inflammatory response, locomotive behavior and defense response found in pGBM, which are critical for protection and progression of tumor cells, while chromosome organization, cell cycle, mRNA processing and mitosis gene sets relating to malignant proliferation of tumor cells were clustered in sGBM. Compared to pGBM, sGBM displayed significant biological progression of malignant transformation and proliferation, which was consistent with the clinical progression of sGBM developed from low-grade glioma.

Overall, these findings demonstrate that the differences between pGBM and sGBM are caused by the molecular subtypes, and highlight the importance of further research into the role of such differences in therapeutic strategies and targeted treatment for pGBM and sGBM.

\section{MATERIALS AND METHODS}

\section{Tumor samples}

A total of 122 GBM samples from the Chinese Glioma Genome Atlas (CGGA) were included in this study, consisting of $88 \mathrm{pGBM}$ and 34 sGBM. Tumor tissue samples were obtained by surgical resection. All pGBM and sGBM cases were defined by two neuropathologists according to the 2007 WHO classification guidelines and Scherer [3]. Only samples with greater than $80 \%$ tumor cells were selected. All samples were obtained by surgical resection before radiation and chemotherapy. All patients provided written informed consent, and the study was approved by the ethics committees of the participating hospitals.

\section{Whole transcriptome sequencing}

Whole transcriptome sequencing was performed as described previously [25, 26]. Briefly, total RNA was isolated from disrupted and homogenized frozen tissue samples using the RNeasy Mini Kit (Qiagen) according to the manufacturer's instructions. A pestle and a QIAshredder (Qiagen) were used to disrupt and homogenize frozen tissue. RNA intensity was checked using 2100 Bioanalyzer (Agilent Technologies) and only high quality samples with an RNA Integrity Number (RIN) value greater than or equal to 7.0 were used to construct the sequencing library. The subsequent steps 
included end repair, adapter ligation, size selection and polymerase chain reaction enrichment. The length of DNA fragment was measured using a 2100 Bioanalyzer, with median insert sizes of 200 nucleotides. The libraries were sequenced on the Illumina HiSeq 2000 platform using the 101-bp pair-end sequencing strategy. Short sequence reads were aligned to the human reference genome $(\mathrm{Hg}$ 19 Refseq) using the Burrows-Wheeler Aligner (BWA, Version 0.6.2-r126) SnpEff software was used to annotate genetic variance $[27,28]$.

\section{Gene set enrichment analysis}

To determine the gene sets related to particular biological processes present in pGBM and sGBM, gene expression profiling and gene set enrichment analysis (GSEA) was performed as described previously [29].

\section{Statistical analysis}

Survival distributions were estimated by KaplanMeier survival analysis, and the log-rank test was used to assess the statistical significance between stratified survival groups using GraphPad Prism 5.0 statistical software. Student's t-test was performed using SPSS 13.0. All data are presented as the mean \pm SE. A two-sided $P$ value $<0.05$ was considered significant.

\section{ACKNOWLEDGEMENTS}

This work was supported by grants from the National High Technology Research and Development Program of China (863) (No.2012AA02A508), National Natural Science Foundation of China (No. 81201993), National Natural Science Foundation of China (No. 81402068), National Natural Science Foundation of China (No.81101901), Jiangsu Province's Key Provincial Talents Program (No.RC2011051).

\section{CONFLICTS OF INTEREST}

All authors report no conflicts of interest relevant to this article.

\section{REFERENCES}

1. Ohgaki H, Kleihues P. Epidemiology and etiology of gliomas. Acta neuropathologica 2005;109(1):93-108.

2. Peiffer J, Kleihues P. Hans-Joachim Scherer (1906-1945), pioneer in glioma research. Brain pathology 1999;9(2):241245.

3. Scherer HJ . Cerebral astrocytomas and their derivatives. Am. J Cancer 1940;40: 159-198.

4. Yan W, Li R, Liu Y, Yang P, Wang Z, Zhang C, Bao Z,
Zhang W, You Y, Jiang T. MicroRNA expression patterns in the malignant progression of gliomas and a 5-microRNA signature for prognosis. Oncotarget 2014;5(24):1290812915.

5. Balss J, Meyer J, Mueller W, Korshunov A, Hartmann C, von Deimling A. Analysis of the IDH1 codon 132 mutation in brain tumors. Acta neuropathologica 2008;116(6):597602 .

6. Watanabe $\mathrm{T}$, Nobusawa $\mathrm{S}$, Kleihues $\mathrm{P}$, Ohgaki $\mathrm{H}$. IDH1 mutations are early events in the development of astrocytomas and oligodendrogliomas. The American journal of pathology 2009;174(4):1149-1153.

7. Yan H, Parsons DW, Jin G, McLendon R, Rasheed BA, Yuan W, Kos I, Batinic-Haberle I, Jones S, Riggins GJ, Friedman H, Friedman A, Reardon D, et al. IDH1 and IDH2 mutations in gliomas. The New England journal of medicine 2009;360(8):765-773.

8. Nobusawa S, Watanabe T, Kleihues P, Ohgaki H. IDH1 mutations as molecular signature and predictive factor of secondary glioblastomas. Clinical cancer research : an official journal of the American Association for Cancer Research 2009;15(19):6002-6007.

9. Killela PJ, Pirozzi CJ, Healy P, Reitman ZJ, Lipp E, Rasheed BA, Yang R, Diplas BH, Wang Z, Greer PK, Zhu H, Wang CY, Carpenter AB, et al. Mutations in IDH1, IDH2, and in the TERT promoter define clinically distinct subgroups of adult malignant gliomas. Oncotarget 2014;5(6):1515-1525.

10. Verhaak RG, Hoadley KA, Purdom E, Wang V, Qi Y, Wilkerson MD, Miller CR, Ding L, Golub T, Mesirov JP, Alexe G, Lawrence M, O'Kelly M, et al. Integrated genomic analysis identifies clinically relevant subtypes of glioblastoma characterized by abnormalities in PDGFRA, IDH1, EGFR, and NF1. Cancer cell 2010;17(1):98-110.

11. Ohgaki H, Kleihues P. Genetic pathways to primary and secondary glioblastoma. The American journal of pathology 2007;170(5):1445-1453.

12. Ohgaki H, Dessen P, Jourde B, Horstmann S, Nishikawa T, Di Patre PL, Burkhard C, Schuler D, Probst-Hensch NM, Maiorka PC, Baeza N, Pisani P, Yonekawa Y, et al. Genetic pathways to glioblastoma: a population-based study. Cancer research 2004;64(19):6892-6899.

13. Ohgaki H, Kleihues P. Population-based studies on incidence, survival rates, and genetic alterations in astrocytic and oligodendroglial gliomas. Journal of neuropathology and experimental neurology 2005;64(6):479-489.

14. Hochberg FH, Linggood R, Wolfson L, Baker WH, Kornblith P. Quality and duration of survival in glioblastoma multiforme. Combined surgical, radiation, and lomustine therapy. Jama 1979;241(10):1016-1018.

15. Watanabe K, Tachibana O, Sata K, Yonekawa Y, Kleihues $\mathrm{P}$, Ohgaki H. Overexpression of the EGF receptor and p53 mutations are mutually exclusive in the evolution of primary and secondary glioblastomas. Brain pathology 
1996;6(3):217-223; discussion 223-214.

16. Houillier C, Wang X, Kaloshi G, Mokhtari K, Guillevin R, Laffaire J, Paris S, Boisselier B, Idbaih A, LaigleDonadey F, Hoang-Xuan K, Sanson M, Delattre JY. IDH1 or IDH2 mutations predict longer survival and response to temozolomide in low-grade gliomas. Neurology 2010;75(17):1560-1566.

17. Ohgaki H, Kleihues P. The definition of primary and secondary glioblastoma. Clinical cancer research : an official journal of the American Association for Cancer Research 2013;19(4):764-772.

18. Li J, Taich ZJ, Goyal A, Gonda D, Akers J, Adhikari B, Patel K, Vandenberg S, Yan W, Bao Z, Carter BS, Wang $\mathrm{R}$, Mao Y, et al. Epigenetic suppression of EGFR signaling in G-CIMP+ glioblastomas. Oncotarget 2014;5(17):73427356.

19. Eustermann S, Yang JC, Law MJ, Amos R, Chapman LM, Jelinska C, Garrick D, Clynes D, Gibbons RJ, Rhodes D, Higgs DR, Neuhaus D. Combinatorial readout of histone H3 modifications specifies localization of ATRX to heterochromatin. Nature structural \& molecular biology 2011;18(7):777-782.

20. Iwase S, Xiang B, Ghosh S, Ren T, Lewis PW, Cochrane JC, Allis CD, Picketts DJ, Patel DJ, Li H, Shi Y. ATRX ADD domain links an atypical histone methylation recognition mechanism to human mental-retardation syndrome. Nature structural \& molecular biology 2011;18(7):769-776.

21. Lewis PW, Elsaesser SJ, Noh KM, Stadler SC, Allis CD. Daxx is an H3.3-specific histone chaperone and cooperates with ATRX in replication-independent chromatin assembly at telomeres. Proceedings of the National Academy of Sciences of the United States of America 2010;107(32):14075-14080.

22. Liu XY, Gerges N, Korshunov A, Sabha N, KhuongQuang DA, Fontebasso AM, Fleming A, Hadjadj D, Schwartzentruber J, Majewski J, Dong Z, Siegel P, Albrecht $\mathrm{S}$, et al. Frequent ATRX mutations and loss of expression in adult diffuse astrocytic tumors carrying IDH1/IDH2 and TP53 mutations. Acta neuropathologica 2012;124(5):615625.

23. Jiao Y, Killela PJ, Reitman ZJ, Rasheed AB, Heaphy CM, de Wilde RF, Rodriguez FJ, Rosemberg S, Oba-Shinjo SM, Nagahashi Marie SK, Bettegowda C, Agrawal N, Lipp E, et al. Frequent ATRX, CIC, FUBP1 and IDH1 mutations refine the classification of malignant gliomas. Oncotarget 2012;3(7):709-722.

24. Lai A, Kharbanda S, Pope WB, Tran A, Solis OE, Peale F, Forrest WF, Pujara K, Carrillo JA, Pandita A, Ellingson BM, Bowers CW, Soriano RH, et al. Evidence for sequenced molecular evolution of IDH1 mutant glioblastoma from a distinct cell of origin. Journal of clinical oncology : official journal of the American Society of Clinical Oncology 2011;29(34):4482-4490.

25. Bao ZS, Chen HM, Yang MY, , Zhang CB, Yu K, Ye WL, Hu BQ, Yan W, Zhang W, Akers J, Ramakrishnan V, Li J,
Carter B, et al. RNA-seq of 272 gliomas revealed a novel, recurrent PTPRZ1-MET fusion transcript in secondary glioblastomas. Genome Research, 2014; 24(11):1765-1773.

26. Cai J, Yang P, Zhang C, Zhang W, Liu Y, Bao Z, Liu X, Du W, Wang H, Jiang T, Jiang C. ATRX mRNA expression combined with IDH1/2 mutational status and Ki-67 expression refines the molecular classification of astrocytic tumors: evidence from the whole transcriptome sequencing of 169 samples samples. Oncotarget 2014;5(9):2551-2561.

27. Li H, Durbin R. Fast and accurate short read alignment with Burrows-Wheeler transform. Bioinformatics 2009;25(14):1754-1760.

28. Cingolani P, Platts A, Wang le L, Coon M, Nguyen T, Wang L, Land SJ, Lu X, Ruden DM. A program for annotating and predicting the effects of single nucleotide polymorphisms, SnpEff: SNPs in the genome of Drosophila melanogaster strain w1118; iso-2; iso-3. Fly 2012;6(2):8092.

29. Subramanian A, Tamayo P, Mootha VK, Mukherjee S, Ebert BL, Gillette MA, Paulovich A, Pomeroy SL, Golub TR, Lander ES, Mesirov JP. Gene set enrichment analysis: a knowledge-based approach for interpreting genomewide expression profiles. Proceedings of the National Academy of Sciences of the United States of America 2005;102(43):15545-15550. 